\title{
Optimum Design of the Lateral Support System for Primary Mirror of Large-aperture Telescope
}

\author{
Yang WANG ${ }^{1, a}$, Jing-Xu ZHANG ${ }^{2, b}$, Xiao-Xia WU ${ }^{2, c}$ \\ ${ }^{1}$ Jilin Agricultural University, Jilin Changchun 130118, China \\ ${ }^{2}$ Changchun Institute of Optis, Fine Mechanics and Physics, Chinese Academy of Sciences, \\ Changchun 130033, China \\ awangyang070611@126.com, 'bhangjx@ciomp.ac.cn, cwu-xiaoxia@sohu.com
}

Keywords: Large Aperture Telescope, Lateral Support, Surface Figure, Finite Element Analysis.

\begin{abstract}
The surface figure precision of the primary mirror in a large-aperture telescope is a key factor to the imaging quality. With the finite-element software, the finite-element model is set up base on optimization of the lateral support system. When the primary mirror is supported in the vertical position, the surface figure deformations of the primary mirror were analyzed. The rigid body displacement is removed, the max deformation of the mirror face is $261 \mathrm{~nm}$, the surface figure deformation RMS value is $64.03 \mathrm{~nm}$, PV value is $251.03 \mathrm{~nm}$. The results may offer some important references for the designs in this area.
\end{abstract}

\section{Introduction}

The support system of the large-aperture telescope is the two parts by the axial and lateral support system. For conventional telescopes, the deformation of the axial support is more sensitive. The mirror surface deformation caused by lateral support is much smaller than the axial support. In the past, we often adopt a lot of lateral support method that could meet the design requirements such as lever balance weight, hydraulic support or steel belt support. With the large of primary mirror aperture, the thickness ratio of the primary mirror becomes much larger. Lateral support design is becoming more and more complicated and important[1-4]. As the altazimuth system, the primary mirror only do pitch movement around the horizontal axis. It is not relevant on the azimuth axis rotation. Therefore, the lateral support of the primary mirror has the directivity. When the primary pointed to the horizontal position, the most weight of the primary mirror is supported by the lateral support. It could play a major role on keep the surface figure error of the primary mirror.

\section{Lateral Support Structure of the Primary Mirror}

There are usually few lateral support methods such as lever counterweight, hydrostatic pressure and steel strap in the past. With increase of diameter to thickness ratio and use of strongly concave mirrors, lateral support is becoming more complicated and important than before. Although the deformation of mirror is usually more sensitive to axial support, the lateral support is a crucial factor to maintain good mirror figure when telescope is used to observe the celestial objects. The mirror lateral support is made from three fixtures located around the mirror periphery and attaching the piece to the telescope structure. These are flexible frames having the shape of the letter A, so they are called "A frame". The "A frame" is stainless steel complex devices linked to the mirror by means of Invar pads glued on the outer ribs. The feet of the "A frame" is attached to the pads through screws in order to allow disassembly for coating and all other maintenance operations. The summit of each"A frame" is fixed on the mirror cell manufactured by MAN.

The lateral support frame includes a counterweight system to minimize deformation of the mirror surface dues to the effects of gravity when pointing away from the zenith. Edge supports involving straps, mercury tubes, push-pull counterweighted levers, springs, and pneumatic/hydraulic actuators are all commonly used in astronomical telescopes, as well as in large-aperture optical systems for other applications. The three typical lateral supports are shown in Figure 1. 


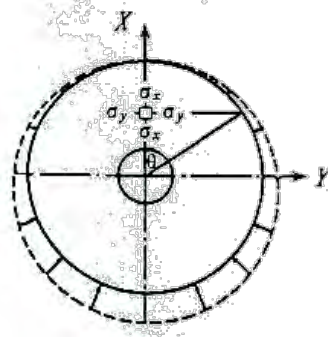

(a)

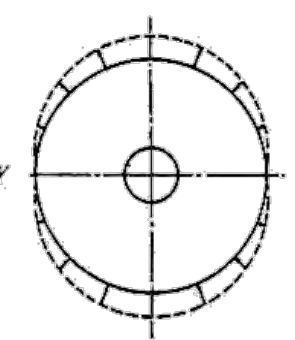

(b)

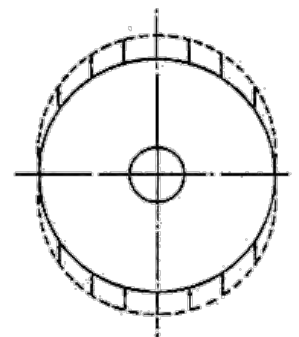

(c)

Fig.1 Sketch of Stress for Several Lateral Supports

(a)the support of mercury straps $\quad \sigma_{y}=-k_{a}(1-\cos \theta) \sin \theta$

(b)the support of radial cosine push $\quad \sigma_{y}=k_{b} \cos \theta \sin \theta$

(c)the support of "push-pull" in vertical direction $\quad \sigma_{y}=0$

In here, $\theta$ is polar angle of lateral support force the polar angle and $k_{a} 、 k_{b}$ are positive constant. We can compare the root mean square (rms) of the primary mirror in three typical lateral supports. Finally, we choose the final project that is optimized by means of the structural analysis software. Ideally, the edge forces exerted by lever counterweight can push up in the lower half of the mirror and pull up on the upper half. The forces should also act in the plane containing the center of gravity of the mirror so that overturning moments are avoided. The most important advantage of lever counterweight is that each support force of floating point can change automatically with variation of direction of gravity, so electric control system is unnecessary. The figure 2(c) shows a push-pull forces scheme, a series of counterweighted radial levers arranged so as to exert parallel push-pull forces on the mirror's edge, the forces are equal in magnitude, support equal-weight vertical slices of the mirror and act in a plane through the mirror's center of gravity.

Push-pull supports are simpler than, for instance, lateral supports, which are internally nested through the back face. They also are more easily adjustable. However, they could only be successfully put into practice after progress in bonding technology permitted safe application to tensile supporting forces. The push-pull principle was later extended to include an additional life by a distribution of tangential shearing forces at the outer edge. Such supports could be designated push-pull-shear supports. It will be shown that the systematic utilization of the shear component, whose potential had not been fully recognized until now, can lead to surprising improvements. The bending distortion of large mirrors can be reduced drastically, in fact, so much that the practical application of such support designs need no longer be effectively limited by mirror size.

\section{Primary Mirror Lateral Support Structure Optimization Analysis}

When the telescope from zenith to levels, namely the zenith Angle is $90^{\circ}$, the weight of the primary mirror completely is supported by the lateral support structure. According to the literatures[5-9], the lateral support design of the primary mirror is adopted by the "A frame" structure. The lateral support distribution diagram is shown in figure 2. The angle $\alpha$ is optimized by using finite element analysis software. When $\alpha$ is $150^{\circ}$, the surface deformation of the primary mirror is best. The three "A frame" stress diagram is shown in figure 2. The lateral support should satisfy the following equation[10]:

$$
\left\{\begin{array}{l}
\sum F_{x}=0, \sum F_{y}=0, \sum F_{z}=0 \\
\sum M_{x}=0, \sum M_{y}=0, \sum M_{z}=0
\end{array}\right.
$$

The primary mirror supporting structure of the large-aperture telescope is mainly based on three tangential lever principles. Each pole is flexible in the bending direction. It does not limit the 
displacement of the primary mirror along the optical axis direction. This equipment is a good coupling device for support axial and lateral support. The lateral support structure way of the primary mirror is shown In figure 3. The "A frame" structure is connected to the primary mirror cell.

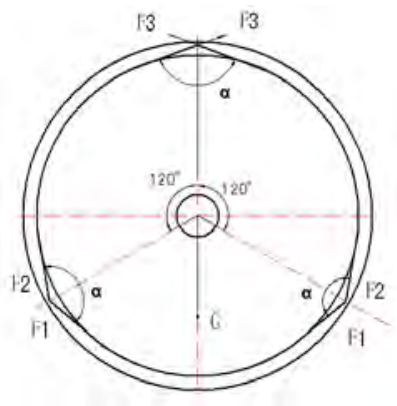

Fig.2 Sketch of "A” bipod lateral supports and stress

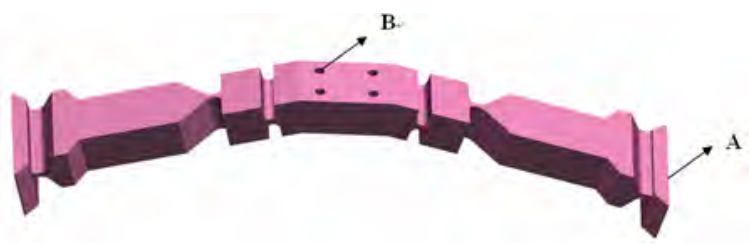

Fig.3 The Structure of "A" Bipod Lateral Supports

The lateral supporting system of the primary mirror mainly consists of the following parts:

(1)Each bipod is a curving stainless steel pole. In order to create bending in four locations, each bipod has a flexible device;

(2)Stainless steel "A" type structure is shown in figure 3. "A" place is connected to the invar pad through screw. The invar pad liner is adhesive on the outer periphery of the primary mirror in order to facilitate coating and disassembly;

(3)The peak of each " $A$ " the structure, namely " $B$ " place will be fixed in the primary mirror cell in figure 3 . Each bipod is rigid on the direction of tangent to the primary mirror side.

In addition, three side support points of the primary mirror cell were translated into six lateral support points of the glass structure through "A frame" structure. That would greatly reduce the weight component that caused the global deformation of the optical surface and local deformation in the primary mirror plane[11-13]. At the same time, the internal stress will be reduced to the half. This is equivalent to increase the intensity of the primary mirror. "A frame" structure would produce the tiny displacement along the optical axis under the lateral support device and would not produce too much additional the edge bending moment.

The figure 4 is the deformation nephogram of "A" bipod lateral support. The maximum stress $\sigma_{\max }$ of "A frame" structure is $34.9 \mathrm{Mpa}$. The allowable stress $[\sigma]$ of the stainless steel materials is $170 \mathrm{Mpa}$. So, $\sigma_{\max }<[\sigma]$ would conform to the design requirements.

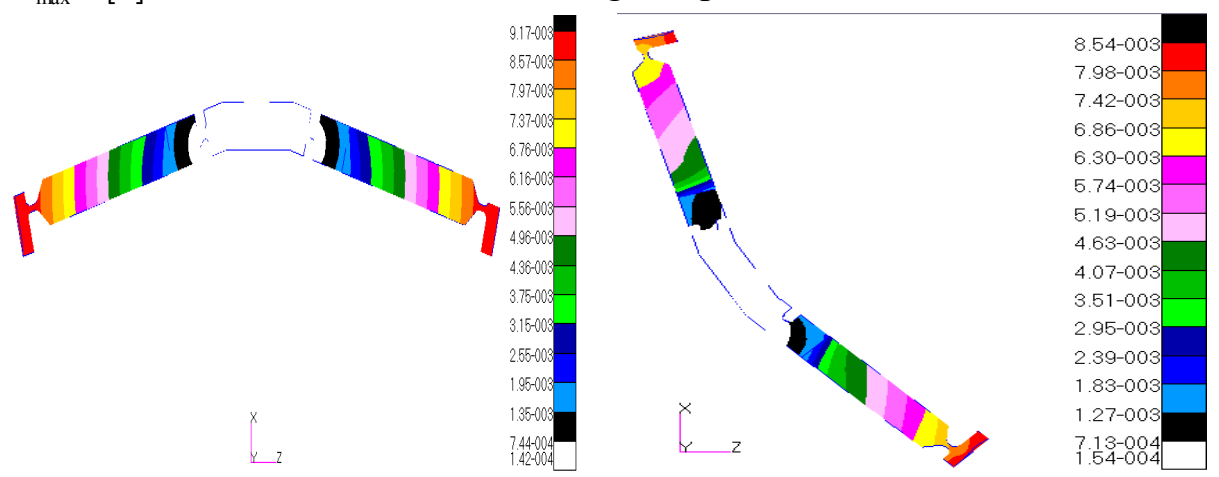

Fig.4 Deformation Nephogram of “A” Bipod Lateral Support

When the primary mirror points to from zenith to horizon, the axial support force is cosine change with zenith angle. Otherwise, the force of the lateral support system is sine change with angle. The axial and lateral support system should be optimized. It could make the optical surface deformation in the zenith angle meet the design requirements. When the optical axis points to the 
level, the whole weight of the mirror is supported by the lateral support. In order to minimize the mirror surface deformation, the resultant force of the lateral support should get through the center of gravity in the primary mirror[14].

In order to quantitatively investigate the surface figure deformation of the primary mirror under the optimization results, the finite element model of the primary mirror was established by the MSC. Patran/Nastran[15]. Each particular support point has the support of the corresponding node. The back 18 points carried through all constraints by the method of manual mesh. The gravity was perpendicular to the optical axis. The results of the optimization analysis were shown in figure 5 . The maximum deformation is $261 \mathrm{~nm}$ after removing of the rigid body displacement. Surface figure error of the RMS value is $64.03 \mathrm{~nm}$. The PV value is $251.03 \mathrm{~nm}$.

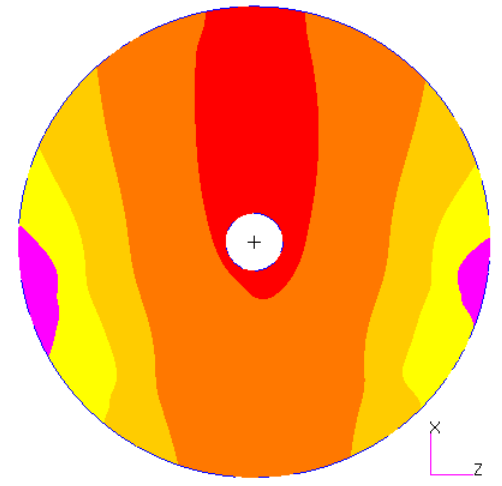

Fig.5 Deformation Nephogram of "A" Bipod Lateral Supports

The optimization of "A frame" structure can greatly reduce mirror surface deformation. By the finite element optimization analysis, the surface figure error of the lateral support the primary mirror components would get the RMS value $(64.03 \mathrm{~nm})$ close to $\lambda / 10(\lambda=632.8 \mathrm{~nm})$. That could basically meet the requirements of the optical design.

\section{Conclusion}

The primary mirror of the large-aperture telescope is the key components in the large telescope. Especially, the support ways of the primary mirror have great influences on the surface figure accuracy. Based on the lateral support forms of the foreign large-aperture telescope primary mirror, we determined the lateral support means of the primary mirror in this paper. The primary mirror is used by three "A frame" bipod lateral support structure with $120^{\circ}$ uniformly distributed on the outer edge of. By means of finite element analysis software ANSYS to complete the side support "A frame" structure optimization analysis. We ultimately determine the bipod angle $\alpha$ is $150^{\circ}$. In the condition of vertical, the mirror surface deformation value is $261 \mathrm{~nm}$. The RMS value is 64.03 $\mathrm{nm}$. The PV value is $251.03 \mathrm{~nm}$. The RMS value is less than $\lambda / 10$ the index requirements. That could satisfy the requirement in the optical design.

\section{References}

[1]CHENG J Q. Astronomical telescope theory and design[M].Beijing: China Science and Technology Press. 2003.(in Chinese)

[2]Paul R. Yoder, Jr. Mounting optics in optical instruments[M]. Bellingham: SPIE Press, 2002.

[3]Xuefei Gong, Xiangqun Cui. Research on mirror lateral support of large astronomical telescope [J]. SPIE, 2006, 6148: 61480Y.

[4]Paul R. Yoder, JR. Opto-mechanical systems design[M]. New York: Marcel Dekker, Inc, 1993.

[5]FU Y, XU C J, DING Y L. Scanning mirror supporting structure of aerial remote sensing 
camera[J]. Optics and Precision Engineering, 2003, 11(6): 550-554.(in Chinese)

[6]NELSON J E. Telescope mirror support s: plate deflections on point supports[J]. SPIE, Vol.332: 212-228.

[7]Jerry E.Nelson, Jacob Lubliner ,Terry S.Mast. Telescope mirror supports: plate deflections on point supports[J]. SPIE, Vol.332: 212-228.

[8]A.J.Malivock. Theoretical elastic deformations of the Steward Observatory $230-\mathrm{cm}$ and the optical sciences center 154-cm mirrors[J]. Applied Optics, 1972, 11(3): 575-585.

[9]Craig Smith, Larry Randall, Ben Greene, et al.Two-meter telescopes for the new millennium[J]. SPIE, Vol.4004: 576-583.

[10]Gong Xuefei, Cui Xiangqun. Research on mirror lateral support of large astronomical telescope[J]. SPIE, Vol.6148:61480Y.

[11]Gordon J.Pentland, Kerry Gonzales, Kevin Harris, et al.The Magdalena ridge observatory $2.4 \mathrm{~m}$ telescope[J]. SPIE, Vol.6267: 62670C-1-62670C-12.

[12]Magomed A.Abdulkadyrov, Sergey P.Belousov Alexandr N.Ignatov, et al.Manufacturing of primary mirrors from Sitall CO-115M for European projects TTL, NOA and VST[J]. SPIE, Vol.4451: 131-137.

[13]A.J.Malivock. Dynamic relaxation: a general method for determination of elastic deformation of mirrors[J]. Applied Optics, 1968, 7(10): 2117-2122.

[14]HE X. The Finite Element Calculation of the Axial Support of a $\phi 220 \mathrm{~cm}$ Primary Mirror[J]. Optics mechanism, 1991, (4): 14-18..(in Chinese)

[15]Alson E. Hatheway. Fine Element Methods for Exaluating Optical System Performance[J]. SPIE, Vol.518: 145-149. 